\title{
Pediatric Melody mitral valve replacement in acute endocarditis: 2 consecutive cases operated-on with an alternative hybrid technique
}

\author{
1 Department of Pediatric Cardiac Surgery, St. Adalbertus Hospital, Gdańsk, Poland \\ 2 Chair of Health and Biological Sciences, Gdańsk Academy of Physical Education and Sport, Gdańsk, Poland \\ 3 Department of Rehabilitation, Medical University of Gdańsk, Gdańsk, Poland
}

Ireneusz Haponiuk ${ }^{1,2}$, Maciej Chojnicki', Konrad Paczkowski', Mariusz Steffens'1, Anna Romanowicz-Sołtyszewska1, Marta Paśko-Majewska1, Monika Opacian-Bojanowska1', Paweł Macko', Julia Haponiuk¹, Katarzyna Gierat-Haponiuk²,3

Correspondence to: Ireneusz Haponiuk, MD, PhD, Department of Pediatric Cardiac Surgery, St. Adalbertus Hospital, Al. Jana Pawła II 50 , 80-462 Gdańsk, Poland, phone: +48587684881 , email: ireneusz_haponiuk@poczta.onet.pl Received: December 9, 2019. Revision accepted: January 10, 2020. Published online: January 10, 2020. Kardiol Pol. 2020; 78 (1): 75-77 doi:10.33963/KP.15132 Copyright by the Author(s), 2020
Introduction Acute endocarditis (AE) is a rare disease in children, presenting with aggressive septic destruction of endocardial structures and consecutive acute heart failure (HF) that necessitates pediatric heart valve surgery in emergency settings. ${ }^{1,2}$ Irreparable valves are a challenge, with a number of limitations related to the lack of prostheses commercially designed for valve replacements in children.

The Melody transcatheter pulmonary valve (Medtronic BV, Heerlen, the Netherlands), a stented bovine jugular vein graft, was introduced for percutaneous implantation in the pulmonary position. Acceptable midterm results were achieved for the alternative implantation technique of mitral valve replacement (MVR) in children..$^{3,4}$ The advantages of the Melody valve are perfect hemodynamics with favorable effective orifice area index, low transannular gradient, intraoperative annular adjustment hybrid strategy, and most importantly, a unique potential for minimally invasive percutaneous balloon dilation following expected growth of the child. ${ }^{5}$

We present a brief report of 2 consecutive pediatric emergency MVRs with the expendable Melody bioprosthesis in a single cardiothoracic surgery center.

Methods The first patient, a 23-month-old girl with a body weight of $12 \mathrm{~kg}$ was referred for surgery due to $\mathrm{HF}$ with critical mitral valve incompetence (MVI) proven by echocardiography and cardiogenic shock. The girl, a premature without a history of congenital heart defects, presented with cardiovascular deterioration after 2 weeks of septicemia with critical decompensation, an incident of cardiopulmonary resuscitation, and a full spectrum of multiorgan failure. Huge antibiotic-resistant vegetations protruding into the mitral valve orifice was confirmed by echocardiography.

The second patient, a 15-month-old boy with a body weight of $8 \mathrm{~kg}$ and a history of misdiagnosed aortic coarctation, after late aortic arch repair at the age of 5 months, initially with left ventricular failure (LVF), and significant MVI. The boy was readmitted to the department because of sepsis and cardiopulmonary deterioration due to mitral AE with positive microbiological tests.

Both children were referred after the progression of congestive HF due to massive MVI and LVF. The girl was treated for pulmonary edema with mechanical ventilation, inotropic support, and high-dose diuretics. The second patient had circulatory instability and multiorgan deterioration despite advanced medical therapy, presented with shortness of breath and peripheral hypoperfusion. In both patients, transthoracic echocardiography (TTE) showed massive MVI with fixed vegetations over the posterior and anterior leaflets, disrupted anterior chordae, left atrial enlargement, and severe LVF. The first blood cultures were all negative and the second, incidentally positive (Staphylococcus warneri in 2 separate blood cultures, additional colonization of Klebsiella pneumoniae).

After 2 weeks of preoperative antibiotic therapy, the children were referred for life-saving 
surgical mitral repair or replacement procedures in emergency settings.

Both 18-mm Melody valves were prepared just before the operations. To enable surgical implantation, sewing cuffs (3-mm stripes of expanded polytetrafluoroethylene [ePTFE], incised every $10 \mathrm{~mm}$; Gore-Tex, W. L. Gore \& Associates Inc., Flagstaff, Arizona, United States) were added externally to the midsection of the stents. The stripes were attached to the stents with interrupted superficial sutures and shallow bites limited to anchor the metal elements. The first Melody valve was prepared with excision of its apical part to avoid any interference with the left ventricular outflow tract (FIGURE 1A). The second Melody valve was double-folded: both ends of the stent were folded outside to shorten the valve and facilitate the implantation in the mitral orifice (FIGURE 1B). In the first patient only, the posterior papillary muscle was fixed with secured sutures and the stent was flared in its atrial part.
Both procedures were performed with extracorporeal circulation, mild hypothermia, and aortic cross-clamp. The mitral valve was exposed via classic transseptal approach. The vegetations of both mitral leaflets were found and dissected. Complete damage of leaflets and chordae was observed. After the gentle resection of vegetations, a small rim of leaflets was left over the annulus to avoid injury to conduction and coronary systems during implantation. Hegar dilators $(14 \times 16 \times 18 \mathrm{~mm})$ were used for initial sizing of the annulus with subsequent local povidone-iodine flush.

The Melody valve prostheses were compressed and crimped to allow the passage through the mitral annulus (FIGURE 1C). The sewing ePTFE ring of the valves was secured to the mitral annulus with 3 semicontinuous sutures, and finally tied immediately after the initial balloon dilatation. Finally, the balloon dilation of the valves was performed with the Tyshak II balloon catheter $16 \mathrm{~mm} / 30 \mathrm{~mm}$ (NuMED Inc, Hopkinton,
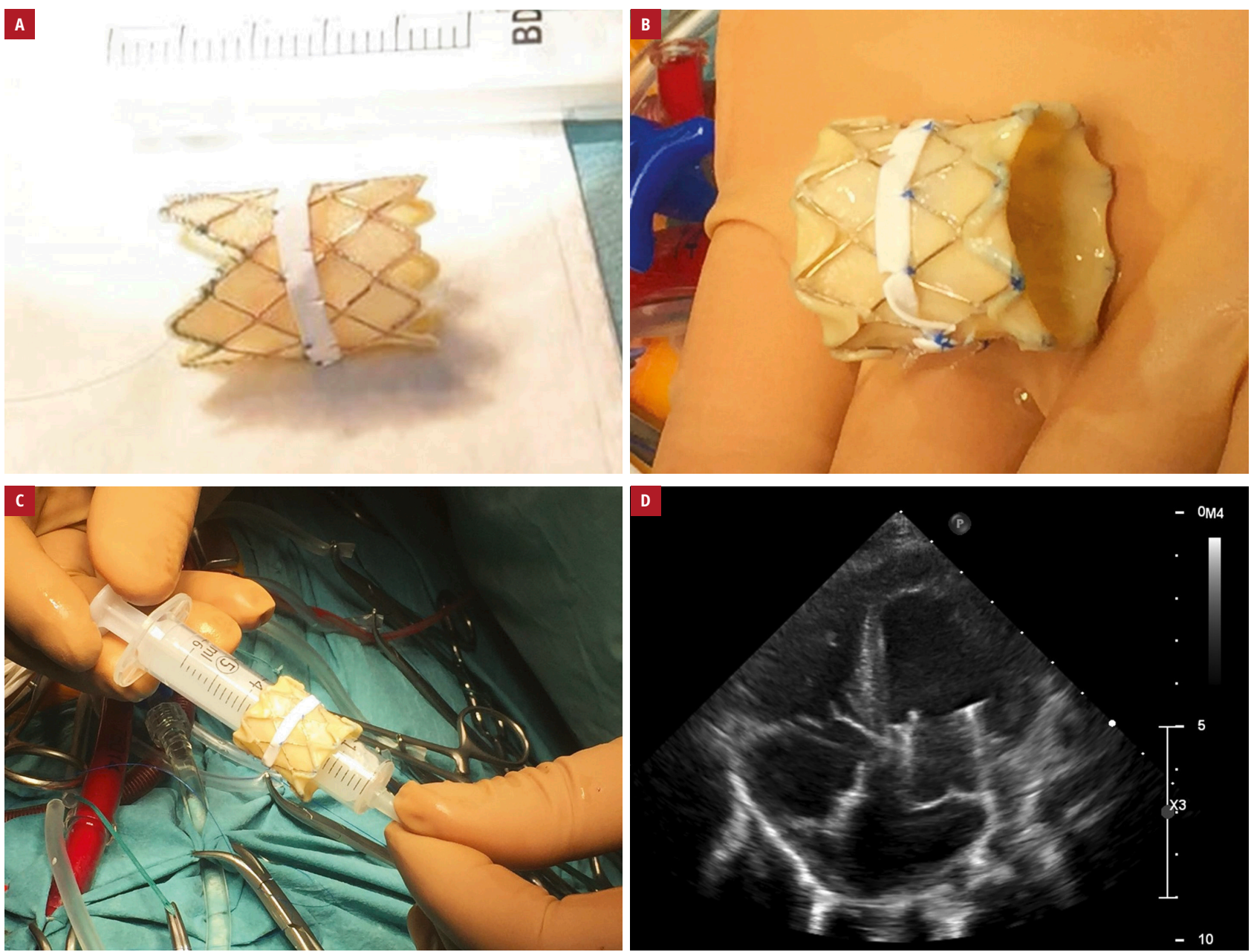

FIGURE 1 A - a Melody valve with its apical part excised to avoid interference with the left ventricular outflow tract. To enable surgical implantation, a sewing 3-mm expanded polytetrafluoroethylene cuff was added externally to the stent. B - a double-folded Melody valve; both ends of the stent were folded outside to shorten the valve. A similar sewing cuff was attached. $\mathbf{C}$ - the Melody valve prostheses were compressed and crimped on a 5 - $\mathrm{cm}$ syringe to allow the passage through the mitral annulus. D - postoperative transthoracic echocardiography showing a good function of Melody MVR without residual paravalvular leaks 
New York, United States) at a pressure of 4 to $6 \mathrm{~atm}$. The balloon sizes were calculated following preoperative measurements of the annular size by echocardiogram and intraoperative Hegar dilator. After dilatation, the valves were inspected to ensure free mobility of all 3 leaflets of the prosthesis. In the first patient, the atrial side of the stent was flared to limit the valve protrusion into the left left atrial cavity, while the second valve was initially double-folded to limit the valvular length. The atrial septal closures with the calibrated ePTFE patch were performed. The epicardial echocardiography and TTE (Philips Epiq 7, Philips Healthcare, Best, the Netherlands) at discharge in both patients confirmed good function of the Melody valves without any signs of left ventricular outflow tract obstruction (FIGURE 1D). Heparin was introduced as postoperative anticoagulation and switched to long-term oral aspirin treatment.

Antibiotics were continued up to 6 weeks in accordance with the European Society of Cardiology guidelines for the treatment of bacterial endocarditis. Our first patient was discharged home after 4 weeks and the second patient, after 6 weeks; both with negative blood cultures and inflammatory parameters within the normal range.

Short- and midterm postoperative follow-up TTEs (2 vs 36 months) showed sustained good competence of the Melody valves. Both children returned back to their normal life activities.

Results and discussion Surgical treatment in children with $\mathrm{AE}$ is still rare; nevertheless, there are some studies reporting successful mitral valve repairs in young children. The authors described mitral valve repair in infants with endocarditis and concluded that mitral valve reconstruction should be the therapy of choice in bacterial endocarditis, particularly in children and adolescents. ${ }^{1}$

There are promising reports of successful Melody MVR procedures in children based on multicenter data. ${ }^{4}$ Although they only include reports on midterm results, there are some significant arguments for the utility of stented expandable bioprosteses for MVRs in small children. They present valid concerns with regard to the length of the Melody valve and the lack of a standard sewing cuff to anchor the device to the atrioventricular annulus. ${ }^{6}$ Therefore, the presented modifications of the valve stents with additionally sewed cuffs and specific implantation techniques are necessary to allow implantation, provide stable position in the mitral annulus, and avoid left ventricular outflow tract obstruction. ${ }^{5}$ In the presented cases, the preparation of the Melody valves was performed before the initiation of the procedure in order to limit the extracorporeal circulation and cross-clamp times.

The reports on the Melody MVR confirmed good function at short- and midterm follow-up, although there is still a limited number of reports on the Melody valves implanted in patients with AE.

The decision to use the Melody valves in 2 consecutive children with $\mathrm{AE}$ were made based on a strong belief that stented bioprostheses may prove more effective than any other artificial valves in the septic environment. The risk of endocarditis associated with the Melody valves was previously reported and analyzed in prostheses implanted in the right ventricular outflow tract and in vitro studies. The risk of infection and damage to the Melody valve exposed to bacteria is similar as in other biological valves and implanted homografts. ${ }^{7,8}$ In line with surgical principles, all potentially dangerous infected tissues were carefully excised and flushed with povidoneiodine before the implantation of the Melody prostheses. The mitral implantation technique with the use of semicontinuous sutures derives from the personal experience of the surgeons.

Conclusions Melody MVR appeared to be a safe and effective treatment for pediatric patients with AE. The acute phase of mitral valve endocarditis was not a contraindication for primary Melody MVR procedures. The Melody valve may be a good solution for the treatment of severe destruction of the mitral valve in children, taking into account the small size of the native mitral ring and the potential for child growth. In this context, it is important to know the technique of valve preparation that ensures its safe surgical implantation.

\section{ARTICLE INFORMATION}

\section{CONFLICT OF INTEREST None declared.}

OPEN ACCESS This is an Open Access article distributed under the terms of the Creative Commons Attribution-NonCommercial-NoDerivatives 4.0 International License (CC BY-NC-ND 4.0), allowing third parties to download articles and share them with others, provided the original work is properly cited, not changed in any way, distributed under the same license, and used for noncommercial purposes only. For commercial use, please contact the journal office at kardiologiapolska@ptkardio.pl.

HOW TO CITE Haponiuk I, Chojnicki M, Paczkowski K, et al. Pediatric Melody mitral valve replacement in acute endocarditis: 2 consecutive cases operated-on with an alternative hybrid technique. Kardiol Pol. 2020; 78: 75-77. doi:10.33963/KP.15132

\section{REFERENCES}

1 Paczkowki K, Chojnicki M, Paczkowska K, et al. Infective endocarditis in children - lasting problem and growing incidence. Folia Cardiologica. 2019; 14: 213- 219.

2 Ammannaya GKK, Sripad N. Fungal endocarditis: what do we know in 2019? Kardiol Pol. 2019; 77: 670-673.

3 Yamaguchi H, Eishi K. Surgical treatment of active infective mitral valve endocarditis. Ann Thorac Cardiovasc Surg. 2007; 13: 150-155.

4 Quinonez LG, Breitbart R, Tworetsky W, et al. Stented bovine jugular vein graft (Melody valve) for surgical mitral valve replacement in infants and children. J Thorac Cardiovasc Surg. 2014; 148: 1443-1449.

5 Pluchinotta FR, Piekarski B, Milani V, et al. Surgical atrioventricular valve replacement with Melody valve in infants and children. A multicenter study. Circ Cardiovasc Interv. 2018; 11: e007145.

6 Emani SM. Melody valve for mitral valve replacement. Operative techniques. Thorac Cardiovasc Surg. 2014; 19: 454-463.

7 Healy DG, Wood AE. Anterior mitral leaflet reconstruction with pericardium in a $1.9 \mathrm{~kg}$ infant with endocarditis. Ann Thorac Surg. 2006; 81: 2310-2312.

8 Jalal Z, Galmiche L, Lebeaux D, et al. Selective propensity of bovine jugular vein material to bacterial adhesions: an in-vitro study. Int J Cardiol. 2015; 198: 201-205. 